\title{
PROGRAMA DE PESQUISA ECOLÓGICA DE LONGA DURAÇÃO - PELD/CNPq - DESAFIOS DA GESTÃO, AVANÇOS E PERSPECTIVAS
}

\author{
Marcia Aparecida Brito ${ }^{1}$, Denise de Oliveira ${ }^{1}$, Marisa de Araujo Mamede ${ }^{2}$, Onivaldo \\ Randig', Fabíla Siqueira de Lacerda ${ }^{1}$
}

\begin{abstract}
${ }^{1}$ Conselho Nacional de Desenvolvimento Científico e Tecnológico, Coordenação do Programa de Pesquisa em Gestão de Ecossistemas, SHIS QI 1 Conjunto B - Bloco A, $1^{\circ}$ andar, Sala 102, CEP: 71605-001, Lago Sul, Brasília, DF, Brasil

${ }^{2}$ Conselho Nacional de Desenvolvimento Científico e Tecnológico, Coordenação Geral do Programa de Pesquisa em Ciências da Terra e do Meio Ambiente, SHIS QI 1 Conjunto B - Bloco A, $1^{\circ}$ andar, Sala 102, CEP: 71605-001, Lago Sul, Brasília, DF, Brasil

E-mails:marcia.brito@cnpq.br (*autor correspondente); denise.oliveira@cnpq.br; marisa.mamede@cnpq.br; onivaldo. randig@cnpq.br; fabiola.lacerda@cnpq.br
\end{abstract}

Resumo: O programa PELD, existente há 23 anos no CNPq, tem sua continuidade assegurada pelo lançamento periódico de Chamadas Públicas e conta atualmente com 34 sítios de referência distribuídos em todos os biomas do país, com atuação em dezenas de unidades de conservação, além de outras áreas protegidas e áreas sujeitas a impactos antrópicos..Possui uma estrutura de gestão bem atuante, na qual os Comitês Gestor e Científico atuam de forma integrada nas decisões estratégicas para o aperfeiçoamento do Programa. É membro da Rede Internacional de Pesquisa Ecológica de Longa Duração - ILTER, que congrega 44 paísesmembros e suas redes de sítios de pesquisa ecológicas de longa duração. O programa vem reunindo esforços para fomentar uma cultura nacional devotada à pesquisa ecológica em torno de questões ambientais e soluções e práticas de uso sustentável dos recursos naturais e dos serviços ecossistêmicos, com importantes contribuições para políticas públicas voltadas à conservação da biodiversidade e o bem-estar humano.

Palavras-chave: financiamento de pesquisa; acompanhamento e avaliação; políticas públicas de ciência e tecnologia; conservação da biodiversidade; governança.

LONG-TERM ECOLOGICAL RESEARCH PROGRAM - PELD/CNPq - MANAGEMENT CHALLENGES, ADVANCES AND PROSPECTS: The PELD program, held for 23 years by CNPq, is maintained by ongoing Public Calls. The PELD network currently has 34 reference sites distributed in all biomes from Brazil, operating in dozens of protected areas and areas subject to anthropic impacts. The Program has a well-established and active management structure, in which the Management and Scientific Committees act in an integrated manner for strategic decisions in order to improve the Program. It is a member of the International Network for Long Term Ecological Research - ILTER, which brings together 44 member countries and their networks of long-term research sites. The program has been pooling efforts to foster a national culture devoted to long-term ecological research that can address environmental issues and point to solutions and practices for sustainable use of natural resources and ecosystem services, with important contributions to the proposition of public policies, promoting biodiversity conservation and human welfare.

Keywords: research funding; monitoring and evaluation; science and technology public policies; biodiversity conservation; governance. 
O Programa de Pesquisas Ecológicas de Longa Duração (PELD) fomenta pesquisas interdisciplinares, buscando compreender a longo prazo os efeitos de perturbações de origens natural e/ou antrópica sobre a composição, dinâmica e funcionamento de ecossistemas, bem como da efetividade de ações de manejo empregadas na sua preservação. O PELD foi criado em 1997 (Brasil 1997, Brasil 1998) sob coordenação do Conselho Nacional de Desenvolvimento Científico e Tecnológico (CNPq), órgão vinculado ao Ministério da Ciência, Tecnologia, Inovações e Comunicações (MCTIC). Desde sua primeira década (1999 - 2009) suas contribuições foram evidentes, do ponto de vista da história natural e do entendimento de processos ecológicos que operam em escalas espaciais e temporais mais amplas (Tabarelli et al. 2013), com subsídios para a gestão ambiental, como exemplo: Agostinho et al. 2004, Rocha et al. 2004 e Seeliger \& Odebrecht 2010.

O avanço científico alcançado pelo Programa tem gerado conhecimentos relevantes sobre a biodiversidade brasileira, estoques de carbono, dinâmica e vulnerabilidade de ecossistemas frente às mudanças do clima e do uso de terra e suas interações. As pesquisas tem subsidiado ações e planos de manejo de Unidades de Conservação (UCs) federais, estaduais e municipais; zoneamento ecológico econômico e identificação de áreas prioritárias para a conservação da biodiversidade (Brasil 2015, Brasil 2019), e contribuído para a consolidação de UCs, como exemplo, o Parque Nacional da Restinga de Jurubatiba, no Rio de Janeiro e o Parque Estadual do Rio Doce, em Minas Gerais (Mamede et al. 2013). Recentemente, os resultados do PELD permitiram elaborar proposta de redefinição da transição Cerrado - Amazônia, com implicações para a conservação da floresta amazônica (Marques et al. 2019).

O CNPq realiza ações de acompanhamento e avaliação do PELD através de um sistema fortalecido pela publicação da Resolução Normativa 023/2011 que estabelece uma estrutura de governança, composta por dois Comitês: Gestor e Científico (Brasil 2011). Com a colaboração da equipe do $\mathrm{CNPq}$, esses comitês vêm contribuindo com importantes inovações ao programa, tais como: (1) ampliação da base de financiamento, com a inclusão da Capes e das Fundações Estaduais de Amparo à Pesquisa (FAPs), fortalecendo a integração com a pós-graduação nacional e a capacidade regional de pesquisa; (2) aumento da visibilidade internacional, através do fortalecimento da inserção na Rede Internacional de Pesquisa Ecológica de Longa Duração - ILTER; (3) normatização da Política de Dados do PELD (Brasil 2016a) e a ampliação da divulgação do programa; (4) explicitação do conceito de pesquisa ecológica de longo prazo, buscando aprimorar a interdisciplinaridade nas pesquisas e fomento à divulgação científica; e (5) cooperação com o Instituto Chico Mendes de Conservação da Biodiversidade (ICMBio), incluindo os gestores ambientais nas equipes de pesquisa.

Atualmente, o PELD é composto por 34 sítios de referência (Figura 1), aprovados na Chamada CNPq/Capes/FAPs/BC-Fundo Newton/PELD ${ }^{\circ}$ 15/2016 (Brasil 2016b), abrangendo pesquisas em ecossistemas sob distintos graus de impacto, distribuídos nos biomas: Amazônia, Mata Atlântica, Caatinga, Cerrado, Pantanal, Campos Sulinos e Bioma Costeiro/Marinho. Apesar disso, permanece ainda a necessidade de ampliar essa rede de sítios visando preencher lacunas geográficas e temáticas, considerando a diversidade ecológica e a magnitude territorial do país.

Os sítios vigentes abordam questões científicas de alta relevância no contexto do desenvolvimento sustentável nacional e internacional, como a investigação e monitoramento dos efeitos das mudanças climáticas sobre a biodiversidade e o funcionamento dos ecossistemas; dos serviços ecossistêmicos; da gestão de ambientes aquáticos de água doce e de ambientes costeiros e marinhos; das invasões biológicas; do impacto e manejo do fogo; da restauração ecológica e recuperação de áreas degradadas.

Pesquisas sobre a avaliação dos impactos ecológicos dos incêndios florestais no bioma Cerrado tem contribuído para a definição de estratégias para a prevenção e o controle do fogo (Miranda 2010), e estudos mais recentes tem demonstrado mudanças na composição e na estrutura da comunidade de pequenos mamíferos devido ao efeito do fogo (Camargo et al. 2018). Vários estudos têm ampliado o conhecimento integrado sobre processos e cadeias produtivas da sociobiodiversidade, como estudos sobre recursos pesqueiros, extrativismo vegetal, turismo ecológico, manejo de pastagens nativas, envolvendo a 


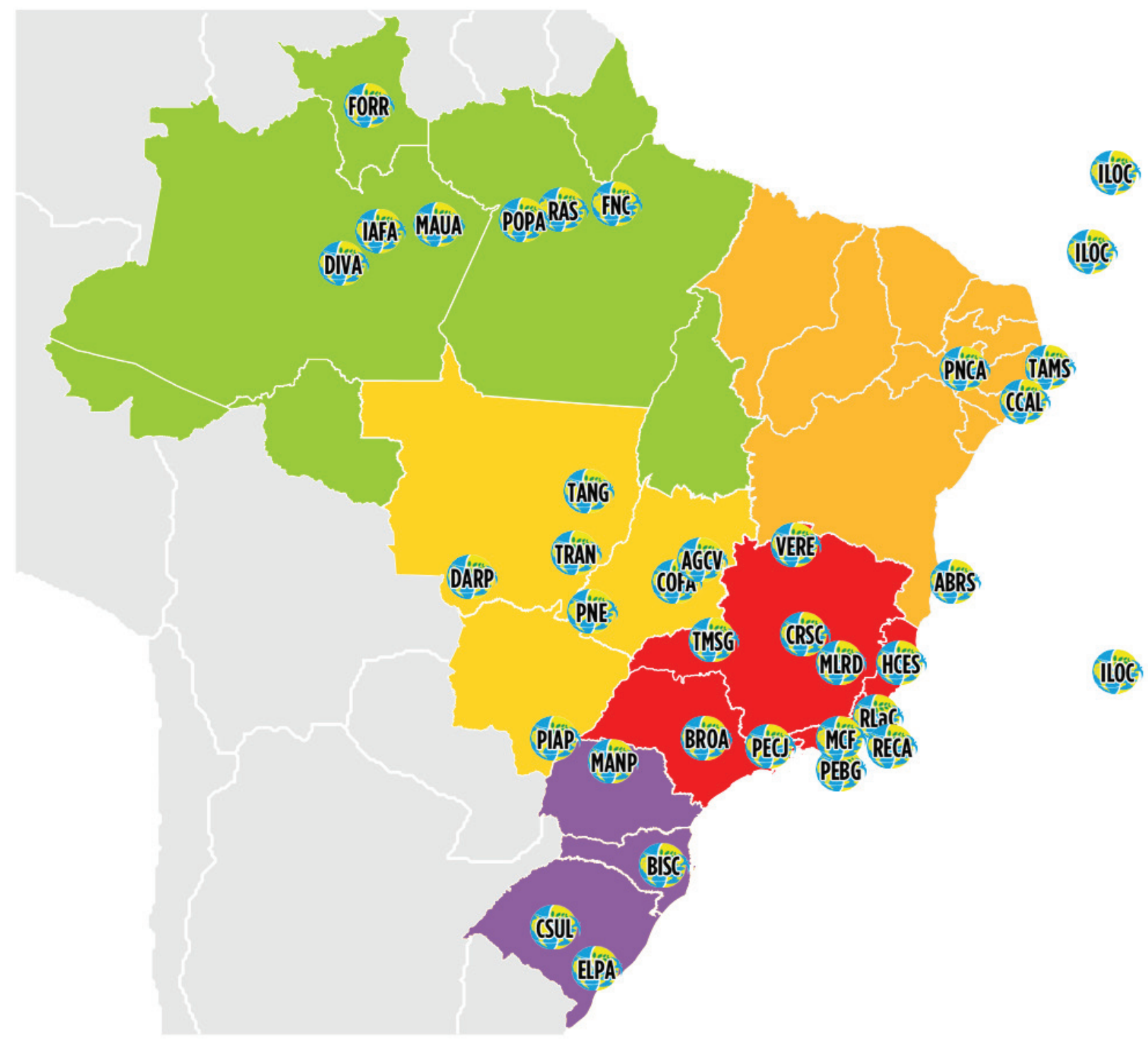

Figura 1. Distribuição dos sítios PELD aprovados na Chamada CNPq/Capes/FAPs/BC-Fundo Newton/ PELD no 15/2016 (vigência: 2016 a 2021). Fonte: http://cnpq.br/sitios-peld/. ABRS - PELD Abrolhos. AGCV - APA Gama Cabeça de Veado, Brasília - DF. BISC - Biodiversidade de Santa Catarina - SC. BROA - Bacias Hidrográficas dos Rios Itaqueri e Lobo e Represa da UHE Carlos Botelho - SP. CCAL - PELD APA Costa dos Corais - AL. CIPÓ - Campos rupestres da Serra do Cipó - MG. COFA - Conectividade Funcional e Antropização na Floresta Nacional de Silvânia - GO. DARP - Dinâmicas Ecológicas na Planície de Inundação do Alto Rio Paraguai - MT. DIVA - Diversidade da Várzea - AM. ELPA - Estuário da Lagoa dos Patos e Costa Adjacente RS. FNC - Floresta Nacional de Caxiuanã - PA. FORR - Florestas de Roraima - RR. HCES - Habitas Costeiros do Espírito Santo - ES. IAFA - Impactos Antrópicos na Floresta Amazônica - Reservas Florestais do INPA - AM. ILOC - Ilhas Oceânicas. MANP - Mata Atlântica do Norte do Paraná. MAUA - PELD Áreas Úmidas Amazônicas - AM. MCF - Mosaico Central Fluminense. MLRD - Mata Atlântica e Sistema Lacustre do médio Rio Doce - MG. PEBG - PELD Baia de Guanabara - RJ. PECJ - Parque Estadual de Campos do Jordão - SP. PELD SUL - PELD Campos Sulinos - RG. PIAP - Planície de Inundação do Alto rio Paraná. PNCA - Parque Nacional do Catimbau - PE. PNE - Parque Nacional das Emas - GO. POPA - PELD do Oeste do Pará - PA. RAS - Rede Amazônia Sustentável - PA. RECA - Ressurgência de Cabo Frio. RLaC - Restingas e Lagoas Costeiras do Norte Fluminense. TAMS - Tamandaré Sustentável - PE. TANG - Fazenda Tanguro - MT. TMSG -Triângulo Mineiro e Sudeste de Goiás. TRAN - Transição Cerrado - Floresta Amazônica - MT. VERE - Veredas no sertão Mineiro - MG. 
valorização do conhecimento tradicional associado (Lopes \& Piedade 2015, Lavrado et al. 2018, Tabarelli et al. 2018, Fischer et al. 2019). O CNPq tem buscado fomentar a divulgação científica em chamadas relacionadas à biodiversidade e, nessa direção, a Chamada PELD no 15/2016 incentivou: (1) produção de conhecimento científico de forma integrada às demandas da sociedade; (2) pesquisa interdisciplinar, interagindo com profissionais das áreas de comunicação e educação; (3) atribuição de um responsável na equipe pela divulgação científica; (4) consideração da pluralidade de públicos a serem integrados à pesquisa (Brasil 2016b).

A avaliação dos relatórios parciais dos 34 projetos dessa Chamada, realizada na $11^{\text {a }}$ Reunião de A\&A, revela avanços quanto à interdisciplinaridade e às ações de divulgação científica. Entretanto, ainda merece atenção o alcance de outros públicos, de forma a abranger também uma escala nacional (Brasil 2019). Almeja-se que a interação com os profissionais das áreas de comunicação e educação possa resultar no aperfeiçoamento de tais ações.

A importância da inserção do componente socioambiental nas pesquisas PELD tem sido destacada desde a criação do Programa, visando uma contribuição mais efetiva para a gestão das UCs e para subsidiar propostas de uso sustentável dos recursos naturais pelas populações locais e regionais (Brasil 1997, Barbosa et al. 2004, Bercini \& Tomanik 2004), em consonância com outras redes internacionais de sítios de pesquisa ecológica de longa duração (Maass et al. 2016, Mirlt et al. 2018).

Em consonância com as metas da Rede ILTER para o engajamento da sociedade na conservação (Kim 2006, Maass et al. 2016, Mirtl et al. 2018), o programa tem buscado promover a conscientização de diversos atores sociais sobre os valores da biodiversidade e dos serviços ambientais, através do incentivo à realização de ações de divulgação científica e educação ambiental. Vale destacar que os sítios PELD tem representado o CNPq nas reuniões nacionais de divulgação e popularização da ciência, como a da Sociedade Brasileira de Progresso da Ciência, realizadas em 2016 e 2017, com exposição dos materiais produzidos pelos sítios PELD Abrolhos e Campos Rupestres da Serra do Cipó. Em 2019, o sítio PELD APA Gama Cabeça deVeado participou da Semana Nacional de Ciência Tecnologia \& Inovação, em Brasília - DF.
Atualmente, 33 sítios PELD abrangem pesquisas em UCs federais, estaduais e/ou municipais, incluindo unidades de proteção integral e de uso sustentável, e apenas 1 (um) sítio está integralmente localizado em área privada, gerando importantes contribuições para a elaboração de planos de manejo dessas UCs.

A partir de uma oficina conjunta $\mathrm{CNPq} / \mathrm{ICMBIO}$ realizada em 2016, ficou evidente a importância do envolvimento dos gestores ambientais nas equipes de pesquisa e da melhoria da comunicação entre esses atores, para atender a demanda de conhecimento científico necessária à gestão das unidades de conservação. Nesse contexto, um desafio para o PELD é fortalecer a integração de ciências sociais e naturais para favorecer o diálogo de saberes.

O PELD/CNPq, ao mesmo tempo em que enfrenta desafios para assegurar sua manutenção e evolução, vem se tornando a principal referência do Brasil em pesquisa em biodiversidade, apresentando grande potencial para 0 desenvolvimento de sínteses do conhecimento, com impactos em políticas públicas para a gestão ambiental e o bem-estar humano, em consonância com outras redes de sítios de longa duração (Maass et al. 2016), na formação de recursos humanos em pesquisas interdisciplinares e com visão de longo prazo e para o alcance das metas nacionais acordadas nas convenções globais do clima, de combate à desertificação e da biodiversidade. Destaca-se nesse contexto a proposta de criação do Centro de Síntese em Biodiversidade e Serviços Ecosssistêmicos - SinBiose (www.sinbiose. cnpq.br), aprovada pelo CNPq em 2017, que se intensificou a partir das discussões ocorridas na reunião de Acompanhamento \& Avaliação que marcou os 20 anos do PELD. Nessa direção, Muelbert et al. (2019) destacam a importância de se realizar sínteses do conhecimento, a partir do potencial dos componentes costeiros e marinhos da Rede ILTER, numa visão global e integrada, visando enfrentar os desafios impostos pelas mudanças climáticas e evolução da ocupação humana na zona costeira.

As perspectivas para o programa abrangem o estímulo às ações conjuntas e sinérgicas entre sítios PELD/CNPq e internacionais (ILTER), para permitir uma compreensão mais abrangente de padrões e processos que afetam a manutenção da biodiversidade em diferentes escalas, bem 
como às ações que intensifiquem a visibilidade e apropriação do conhecimento gerado pelo Programa PELD pela sociedade brasileira, o que demanda uma estratégia de comunicação e divulgação valorizando a conexão entre as pesquisas realizadas e sua importância para a sociedade.

Considerando a inquestionável relevância de um programa de pesquisas de longa duração para o Brasil, a legitimidade do PELD como vetor de geração de conhecimento socialmente relevante sobre os nossos ecossistemas e a capacidade de agregar parceiros demonstrada pelo Programa em sua evolução, os recursos financeiros atualmente disponíveis são insuficientes para o alcance dos muitos objetivos propostos. Assim, o principal desafio que se coloca para o PELD é assegurar um orçamento anual consistente e condizente com o tamanho dos desafios e responsabilidades necessários a um Programa de Pesquisa Ecológica de Longa Duração amplo, robusto e conectado com as questões atuais da sociedade.

\section{REFERÊNCIAS}

Agostinho, A. A., Rodrigues, L., Gomes, L. C., Thomaz, S. M., \& Miranda, L. E. (Eds.). 2004. Structure and functioning of the Paraná River and its floodplain: LTER - site 6 - (PELD - Sítio 6). Maringá: EDUEM. p. 275.

Barbosa, F. A. R., Scarano, F.R., Sabará, M.G., \& Esteves, F. A. 2004. Brazilian LTER: ecosystem and biodiversity information in support of decision-making. Environmental Monitoring and Assessment 90 (1-3), 121-133.

Bercini, L. O., \& Tomani, E.A. 2004. Health, Environment and Social Representations in the Population of Puerto Rico, Paraná State. Em: A. A. Agostinho, L. Rodrigues, L. C. Gomes, S. M. Thomaz, \& L. E. Miranda, (Eds.). Structure and functioning of the Paraná River and its floodplain: LTER - site 6 - (PELD - Sítio 6). pp 265-269. Maringá: EDUEM.

Brasil.1997.Conselho NacionaldeDesenvolvimento Científico e Tecnológico. 1997. Manual Operativo do Programa Integrado de Ecologia. Brasília, DF. 26p.

Brasil. 1998. Conselho Nacional de Desenvolvimento Científico e Tecnológico - CNPq. Programa Integrado de Ecologia -
PIE. Resolução Normativa 016/1998, de 17 de dezembro de 1998. Brasília, DF. (Retrieved on February 7rd, 2020, from http://portalintranet.cnpq.br/web/instrumentos-legais/ normas?p_p_id=novaintranetportlet_ WAR_novaintranetnormasportlet

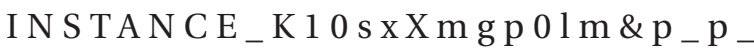
lifecycle $=0 \& p_{-} p_{-}$state $=$normal $\& p_{-} p_{-}$ mode $=$ view $\&$ p_p_col_id $=$ column $-3 \&$ p_p_col_ count=1\&norma=view\&idNorma=617621).

Brasil.2011.ConselhoNacionaldeDesenvolvimento Científico e Tecnológico. Política de Dados do Programa de Pesquisa Ecológica de Longa Duração - PELD. Resolução Normativa 023/2011, de 08 de setembro de 2011. Brasília, DF. (Retrieved on February 7rd, 2020, from http:// portal-intranet.cnpq.br/web/instrumentoslegais/normas?p_p_id=novaintranetportlet_ WAR_novaintranetnormasportlet

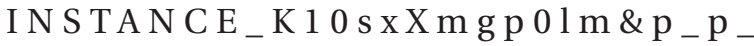
lifecycle $=0 \& p_{-} p_{-}$state $=$nor mal $\& p_{-} p_{-}$ mode=view\&p_p_col_id=column-3\&p_p_col_ count $=1$ \&norma $=$ view\&idNorma $=565332$ )

Brasil.2015.ConselhoNacionaldeDesenvolvimento Científico e Tecnológico - CNPq. Relatório do Comitê Científico: Avaliação do PELD Programa de Pesquisa Ecológica de Longa Duração. 9a Reunião de Acompanhamento \& Avaliação do Programa de Pesquisa Ecológica de Longa Duração - PELD. Relatório Digitalizado. Brasília, DF. 3p.

Brasil. 2016a. Conselho Nacional de Desenvolvimento Científico e Tecnológico. Política de Dados do Programa de Pesquisa Ecológica de Longa Duração - PELD. Resolução Normativa 009/2016, de 13 de abril de 2016. Brasília, DF. (Retrieved on February 3rd, 2020, from https://tinyurl.com/ybtyqkmj).

Brasil. 2016b. Conselho Nacional de Desenvolvimento Científico e Tecnológico. Chamada CNPq/Capes/FAPs/BC-Fundo Newton/PELD no 15/2016 - Pesquisa Ecológica de Longa Duração, de 01 de agosto de 2016. Brasília, DF. (Retrieved on February 3rd, 2020, from https://tinyurl.com/y8lkqug7).

Brasil.2019.ConselhoNacionaldeDesenvolvimento Científico e Tecnológico - CNPq. Relatório do Comitê Científico: Avaliação do PELD Programa de Pesquisa Ecológica de Longa Duração. 11 $1^{\text {a }}$ Reunião de Acompanhamento \& 
Avaliação do Programa de Pesquisa Ecológica de Longa Duração - PELD. Relatório Digitalizado. Brasília, DF. 3p.

Camargo, A. C. L., Barrio, R. L., Camargo, N. F., Mendonça, A. F., Ribeiro, J. F, Rodrigues, C. M. F., \& Vieira, E. M. 2018. Fire affects the occurrence of small mammals at distinct spatial scales in a neotropical savanna. European Journal of Wildlife Research, 64, 1-11. https://doi. org/10.1007/s10344-018-1224-8

Fischer, F.M., Bonet, O., Cezimbra, I., \& Pillar, V.D. 2019. Long-term effects of grazing pressure on functional strategies and components of diversity in subtropical grassland. Applied Vegetation Science 22 (1), 39-47.

Kim, E. 2006. Development, potentials, and challenges of the International Long-Term Ecological Research (ILTER) Network. Ecological Research, 21, 788-793. DOI 10.1007/s11284006-0049-1

Lavrado, P. L., Carvalho, C. de, Keunecke, K. A., \& Vianna, M. 2018. Recursos Vivos: peixes e crustáceos. In: Valentin, J. L., Paiva, P. C. de, \& Salomon, P.S. (Orgs.). A Baía de Guanabara: Passado, presente e futuro de um ecossistema ameaçado. pp. 277-286. Curitiba, PR: CRV.

Lopes, A., \& Piedade, M. T. F. 2015. Conhecendo as áreas úmidas amazônicas: uma viagem pelas várzeas e igapós. Manaus: editora INPA: p. 164.

Maass, M., Balvanera, P., Bourgeron, P., Equihua, M., Baudry, J., Dick, J., Forsius, M., Halada, L., Krauze, K., Nakaoka, M., Orenstein, D. E., Parr, T. W., Redman, C. L., Rozzi, R., Santos-Reis, M., Swemmer, A. M., \& Vădineanu, A. 2016. Changes in biodiversity and trade-offs among ecosystem services, stakeholders, and components of wellbeing: the contribution of the International Long-Term Ecological Research network (ILTER) to Programme on Ecosystem Change and Society (PECS). Ecology and Society 21(3),1-14. http://dx.doi.org/10.5751/ES-08587-210331

Mamede, M. de A., Pinheiro, F. da C., \& Tabarelli, M. 2013. Dez anos de Gestão do PELD: resultados, lições e perspectivas. In: Tabarelli, M.; Rocha, C. F. D.; Romanowski, H. P.; Rocha, O.; Lacerda, L. D. (Eds). PELD - CNPq. Dez anos do Programa de Pesquisas Ecológicas de Longa Duração no Brasil, Achados, Lições e Perspectivas. pp. 415-436. Recife, BR: Editora Universitária da Universidade Federal de Pernambuco.
Marques, E. Q., Marimon-Junior, B.H., Marimon, B.S., Matricardi, E. A. T., Mews, H. A., \& Colli, G. R. 2019. Redefining the Cerrado-Amazonia transition: implications for conservation. Biodiversity and Conservation. Biodiversity and Conservation, 5, 1-17. https://doi.org/10.1007/ s10531-019-01720-z

Miranda, H. S. 2010. Efeitos do regime de fogo sobre a estrutura de comunidades de Cerrado: resultados do projeto Fogo. Brasília - DF: IBAMA/MMA: p. 144.

Mirtl, M., Borer, E. T., Djukic, I., Forsius, M., Haubold, H., Hugo, W., Jourdan, J., Lindenmayer, D., McDowell, W.H., Muraoka, H., Orenstein, D.E., Pauw, J.C., Peterseil, J., Shibata, H., Wohner, C., Yu, X., \& Haase, P. 2018. Genesis, goals and achievements of Long-Term Ecological Research at the global scale: a critical review of ILTER and future directions. Science of the Total Environment 626, 1439-1462. https://doi. org/10.1016/j.scitotenv.2017.12.001

Muelbert, J. H., Nidzieko, N. J., Acosta, A. T. R., Beaulieu, S. E., Bernardino, A. F, Boikova, E. Bornman, T. G., Cataletto, B., Deneudt, K., Eliason, E., Kraberg, A., Nakaoka, M., Pugnetti, A., Ragueneau, O., Scharfe, M., Soltwedel, T., Sosik, H. M., Stanisci, A., Stefanova, K., Stéphan, P., Stier, A., Wikner, J., \& Zingone, A. ILTER - The International Long-Term Ecological Research Network as a Platform for Global Coastal and Ocean Observation. Frontiers in Marine Science, 6, 527. https://doi.org/10.3389/ fmars.2019.00527

Rocha, C. F. D., Esteves, F. A., \& Scarano, F. R. 2004. Pesquisas de longa duração na Restinga de Jurubatiba: ecologia, história natural e conservação. São Carlos: RiMa. p. 376

Seeliger, U., \& Odebrecht, C. 2010. O estuário da Lagoa dos Patos: um século de transformações. Rio Grande: FURG. p. 180

Tabarelli, M., Rocha, C. F. D., Barbosa, F. A. R., Hay, J. D.; Romanowski, H. P., Rocha, O., \& Lacerda, L. D. 2013. Dez anos de PELD: Achados Científicos e Perspectivas. In: Tabarelli, M., Rocha, C. F. D., Romanowski, H. P, Rocha, O., \& Lacerda, L. D. (Editores). PELD - CNPq: Dez anos do Programa de Pesquisas Ecológicas de Longa Duração no Brasil: Achados, Lições e Perspectivas. pp. 396413 Recife: Ed. Universitária da UFPE.

Tabarelli, M., Leal, I. R., Scarano, F. R., \& Silva, J. 
2018. Caatinga: legado, trajetória e desafios rumo à sustentabilidade. Ciência e Cultura, 70 (4), 25-29.

Submitted: 3 September 2019

Accepted: 17 February 2020

Published on line: 15 June 2020

Associate Editors: Camila Barros and Nuria Pistón 